PUBLICATIONS DE L'INSTITUT MATHÉMATIQUE

Nouvelle série, tome 74(88) (2003), 37-56

\title{
MEAN VALUE OF PILTZ' FUNCTION OVER INTEGERS FREE OF LARGE PRIME FACTORS
}

\author{
Servat Nyandwi
}

Communicated by Aleksandar Ivić

\begin{abstract}
We use the saddle-point method (due to Hildebrand-Tenenbaum [3]) to study the asymptotic behaviour of $\sum_{n \leqslant x, P(n) \leqslant y} \tau_{k}(n)$ for any $k>0$ fixed, where $P(n)$ is the greatest prime factor of $n$ and $\tau_{k}$ is Piltz' function. We generalize all results in [3], where the case $k=1$ has been treated.
\end{abstract}

\section{Introduction}

Let $f(n)$ be a multiplicative function. It seems interesting to investigate the mean value of $f(n)$ over integers free of large prime factors, i.e. to study the asymptotic behaviour of

$$
S_{f}(x, y):=\sum_{n \leqslant x, P(n) \leqslant y} f(n)
$$

in domain of $(x, y)$ as large as possible, where $P(n)$ is the greatest prime factor of the integer $n>1$ with the convention $P(1)=1$. The most interesting case is $f(n)=\mathbf{1}(n) \equiv 1$. As usual we write $\Psi(x, y)$ for $S_{\mathbf{1}}(x, y)$, which is the number of positive integers $\leqslant x$ and free of prime factors $>y$. This function appears in diverse areas of number theory and has been received much attention. For a detailed description, we refer the reader to two excellent surveys ([5], [4]). Here we only mention two main results on $\Psi(x, y)$.

In the sequel, we set systematically $u:=\log x / \log y$ and use $\varepsilon$ to denote a sufficiently small positive number. Let $\rho(u)$ be the Dickman function, i.e. the unique continuous solution of the differential-difference equation

$$
u \rho^{\prime}(u)=-\rho(u-1) \quad(u>1), \quad \rho(u)=1 \quad(0 \leqslant u \leqslant 1) .
$$

2000 Mathematics Subject Classification. Primary 11N25. 
By introducing a new type of identity, Hildebrand [2] has proved that the asymptotic formula

$$
\Psi(x, y)=x \rho(u)\left\{1+O\left(\frac{\log (2 u)}{\log y}\right)\right\}
$$

holds uniformly in the range

$$
x \geqslant 3, \quad \exp \left\{(\log \log x)^{5 / 3+\varepsilon}\right\} \leqslant y \leqslant x .
$$

The error term in (1.2) is best-possible and the lower limit in the range $\left(H_{\varepsilon}\right)$ is the limit of what can be reached unconditionnally. In fact Hildebrand [1] has shown that (1.2) in the form $\Psi(x, y)=x \rho(u) \exp \{O(\log (2 u) / \log y)\}$ holds uniformly in the range $y \geqslant 2,1 \leqslant u \leqslant y^{1 / 2-\varepsilon}$, if and only if the Riemann Hypothesis is true.

In aim of seeking estimate for $\Psi(x, y)$ in a larger range, Hildebrand and Tenenbaum [3] have introduced a new method. They start from the Perron formula and use the saddle-point method in the process of estimating the complex integral. This method has many other applications and is now known in analytic number theory under the title of the saddle-point method. For an excellent description on this method, we refer the reader to the paper of Tenenbaum [9]. Applying the saddle-point method, they have obtained an approximation for $\Psi(x, y)$ uniformly for all $x \geqslant y \geqslant 2$ and some short interval results for $\Psi(x, y)$. Define

$$
\begin{array}{lll}
\zeta(s, y):=\prod_{p \leqslant y}\left(1-p^{-s}\right)^{-1}, & \bar{u}:=\min \left\{u, \frac{y}{\log y}\right\} \\
\varphi(s, y):=\log \zeta(s, y), & \varphi_{l}(s, y):=\frac{d^{l}}{d s^{l}} \varphi(s, y) .
\end{array}
$$

Let $\alpha(x, y)$ be the unique positive solution to the equation $\log x+\varphi_{1}(\alpha, y)=0$. Thus the main result of Hildebrand and Tenenbaum can be stated as follows: one has uniformly for $x \geqslant y \geqslant 2$,

$$
\Psi(x, y)=\frac{x^{\alpha} \zeta(\alpha, y)}{\alpha \sqrt{2 \pi \varphi_{2}(\alpha, y)}}\left\{1+O\left(\frac{1}{\bar{u}}\right)\right\}
$$

which yields an symptotic formula whenever $u, y \rightarrow \infty$.

Another interesting multiplicative function is the Piltz function $\tau_{z}(n)(z \in \mathbb{C})$, defined by

$$
\zeta(s)^{z}=\sum_{n=1}^{\infty} \frac{\tau_{z}(n)}{n^{s}} \quad(\operatorname{Re} s>1),
$$

where $\zeta(s)$ is the Riemann function. Clearly $\tau_{z}(n)$ is a natural generalization of $\mathbf{1}(n)(z=1)$ and of $k$-multiple divisor function $\tau_{k}(n)=\mathbf{1} * \tau_{k-1}(n)(z=k \in \mathbb{N})$. For simplicity, we write $S_{z}(x, y)$ for $S_{\tau_{z}}(x, y)$. For any $k>0$ fixed, Smida [8] has shown that one can adapt the saddle-point method to deal with $S_{k}(x, y)$. By using 
the saddle-point method in the version of Saias [6] with some new ideas, she [8] has proved, in the range $\left(H_{\varepsilon}\right)$,

$$
S_{k}(x, y)=x(\log y)^{k-1} \rho_{k}(u)\left\{1+O\left(\frac{\log (2 u)}{\log y}+\frac{1}{(\log y)^{k}}\right)\right\}
$$

where $\rho_{k}(u)$ is the unique continuous solution of the differential-difference equation

$$
\begin{aligned}
u \rho_{k}^{\prime}(u) & =(k-1) \rho_{k}(u)-k \rho_{k}(u-1), & & \text { if } u>1, \\
\rho_{k}(u) & =u^{k-1} / \Gamma(u), & & \text { if } 0<u \leqslant 1,
\end{aligned}
$$

and $\Gamma(u)$ is the usual $\Gamma$-function. Note that, in the case of integer values of $k$, Xuan [12] has obtained the same formula in the same range, by induction on $k$. Obviously (1.4) contains Hildebrand's result (1.2).

The aim of this paper is to apply the method of Hildebrand-Tenenbaum [3] to investigate $S_{k}(x, y)$ for any $k>0$ fixed as in [8]. This work seems interesting: On the one hand we could give a complementary study on $S_{k}(x, y)$ and, on the other hand we could generalize the results of [3]. Before stating our results, we first introduce some notations. Define

$$
\begin{aligned}
L_{\varepsilon}(y) & :=\exp \left\{(\log y)^{3 / 5-\varepsilon}\right\}, & Y_{\varepsilon}(y) & :=\exp \left\{(\log y)^{3 / 2-\varepsilon}\right\} \\
\phi(s, y) & :=k \log \zeta(s, y), & \phi_{l}(s, y) & :=\frac{d^{l}}{d s^{l}} \phi(s, y) .
\end{aligned}
$$

For $u>1$, let $\xi(u)$ be the unique real nonzero root of the equation $e^{\xi(u)}=1+u \xi(u)$. By convention, we set $\xi(1)=0$. We put $\xi:=\xi(u / k)$. For $s \in \mathbb{C}$, we define

$$
I(s):=\int_{0}^{s} \frac{e^{v}-1}{v} d v, \quad \sigma_{j}:=k I^{(j)}(\xi) \quad\left(j \in \mathbb{Z}^{+}\right) .
$$

Let $\alpha_{k}(x, y)$ be the unique positive solution to the equation $\log x+\phi_{1}\left(\alpha_{k}, y\right)=0$. Finally we use $c_{i}=c_{i}(k)$ to denote some positive constants depending on $k$ only. The constants implied in the symbols $O, \ll, \asymp$ depend on $\varepsilon, k$ at most.

Our main result is as follows.

Theorem 1. Let $k>0$ be fixed. For $x \geqslant y \geqslant 2$, we have

$$
S_{k}(x, y)=\frac{x^{\alpha_{k}} \zeta\left(\alpha_{k}, y\right)^{k}}{\alpha_{k} \sqrt{2 \pi \phi_{2}\left(\alpha_{k}, y\right)}}\left\{1+O\left(\frac{1}{\bar{u}}\right)\right\}
$$

This yields an asymptotic formula whenever $u, y \rightarrow \infty$.

The next Theorem 2 gives a smooth approximation for the main term in Theorem 1. 
THEOREM 2. Let $k>0$ be fixed.

(i) For $x \geqslant y \geqslant 2$, we have

$$
\begin{aligned}
& \alpha_{k}(x, y)=\frac{\log (1+y / \log x)}{\log y}\left\{1+O\left(\frac{\log \log y}{\log y}\right)\right\}, \\
& \phi_{2}\left(\alpha_{k}, y\right)=\left(1+\frac{\log x}{y}\right) u(\log y)^{2}\left\{1+O\left(\frac{1}{\log (2 u)}+\frac{1}{\log y}\right)\right\} .
\end{aligned}
$$

(ii) For $y \geqslant 2$ and $1 \leqslant u \leqslant y^{1-\varepsilon}$, we have

$$
\frac{x^{\alpha_{k}} \zeta\left(\alpha_{k}, y\right)^{k}}{\alpha_{k} \sqrt{2 \pi \phi_{2}\left(\alpha_{k}, y\right)}}=\frac{x(\log y)^{k-1}}{\sqrt{2 \pi \sigma_{2}}} e^{k \gamma+\sigma_{0}-u \xi+O\left(u / L_{\varepsilon}(y)+\log (2 u) / \log y\right)},
$$

where $\gamma$ is the Euler constant. Further in the range $\left(H_{\varepsilon}\right)$, we have

$$
\frac{x^{\alpha_{k}} \zeta\left(\alpha_{k}, y\right)^{k}}{\alpha_{k} \sqrt{2 \pi \phi_{2}\left(\alpha_{k}, y\right)}}=x(\log y)^{k-1} \rho_{k}(u)\left\{1+O\left(\frac{1}{u}+\frac{u}{L_{\varepsilon}(y)}+\frac{\log (2 u)}{\log y}\right)\right\} .
$$

Combining Theorem 1 and Theorem 2(i), we immediately obtain the following corollary, which shows that the behaviour of $S_{k}(x, y)$ has a radical change as $y / \log x \rightarrow 0$ or $y / \log x \rightarrow \infty$.

Corollary 1. Let $k>0$ be fixed. For $x \geqslant y \geqslant 2$, we have

$$
S_{k}(x, y)=\frac{x^{\alpha_{k}} \zeta\left(\alpha_{k}, y\right)^{k}}{\sqrt{2 \pi u(1+\log x / y)} \log (1+y / \log x)}\left\{1+O\left(\frac{1}{\log (2 u)}+\frac{1}{\log y}\right)\right\} .
$$

In particular we have

$$
\begin{aligned}
& S_{k}(x, y) \sim \frac{x^{\alpha_{k}} \zeta\left(\alpha_{k}, y\right)^{k}}{\sqrt{2 \pi y / \log y}} \quad(y / \log x \rightarrow 0, y \rightarrow \infty), \\
& S_{k}(x, y) \sim \frac{x^{\alpha_{k}} \zeta\left(\alpha_{k}, y\right)^{k}}{\sqrt{2 \pi u} \log (y / \log x)} \quad(y / \log x \rightarrow \infty, u \rightarrow \infty) .
\end{aligned}
$$

From Theorem 1, we can derive the following simple formula, which describes the local behaviour of $S_{k}(x, y)$ quite precisely.

Theorem 3. Let $k>0$ be fixed. For $x \geqslant y \geqslant 2$ and $1 \leqslant r \leqslant y$, we have

$$
S_{k}(r x, y)=S_{k}(x, y) r^{\alpha_{k}(x, y)}\{1+O(1 / \bar{u})\} .
$$

Combining Theorem 3 and (1.6), we easily get the following result.

Corollary 2. Let $k>0$ be fixed and let $u, y \rightarrow \infty$. Then $S_{k}(2 x, y) \sim S_{k}(x, y)$ if and only if $\log y \leqslant\{1+o(1)\} \log \log x$, and $S_{k}(2 x, y) \sim 2 S_{k}(x, y)$ if and only if $\log y / \log \log x \rightarrow \infty$.

Finally we prove a short interval result on $S_{k}(x, y)$. 
Theorem 4. Let $k>0$ be fixed. For $x \geqslant y \geqslant 2$ and $z \geqslant 1$, we have

$S_{k}(x+x / z, y)-S_{k}(x, y)=\frac{\alpha_{k}(x, y)}{z} S_{k}(x, y)\left\{1+O\left(\frac{1}{z}+\frac{1}{\bar{u}}\right)\right\}+O\left(S_{k}(x, y) R(x, y)\right)$, where $R(x, y):=Y_{\varepsilon}(y)^{-1}+e^{-c_{1} u /(\log 2 u)^{2}} \log y$.

By Theorem 4 and (1.6), we easily obtain the following result.

Corollary 3. Let $k>0$ be fixed. For $x \geqslant 2,(\log \log x)^{2 / 3+\varepsilon} \leqslant \log y \leqslant$ $(\log x)^{2 / 5}$ and $z \leqslant Y_{\varepsilon}(y)$, we have

$$
S_{k}(x+x / z, y)-S_{k}(x, y)=\frac{\log (1+y / \log x)}{z \log y} S_{k}(x, y)\left\{1+O\left(\frac{1}{z}+\frac{\log \log y}{\log y}\right)\right\} .
$$

\section{Technical preparation}

This section is devoted to establishing some preliminary lemmas, which will be needed in the proofs of Theorems 1-4.

LEMma 2.1. Let $k>0$ be fixed. We have uniformly for $y \geqslant 2$ and $\sigma>0$,

$$
\begin{aligned}
-\phi_{1}(\sigma, y) & =\left\{1+O\left(\frac{1}{\log y}\right)\right\} \frac{k}{1-y^{-\sigma}} \int_{1}^{y} \frac{d t}{t^{\sigma}}+O(1), \\
\phi_{2}(\sigma, y) & =\left\{1+O\left(\frac{1}{\log y}\right)\right\} \frac{k}{\left(1-y^{-\sigma}\right)^{2}} \int_{1}^{y} \frac{\log t}{t^{\sigma}} d t+O(1) .
\end{aligned}
$$

Moreover, for any fixed positive constants $\varepsilon$ and $\sigma_{0}$, the error terms $O(1 / \log y)$ can be replaced by $O_{\varepsilon, \sigma_{0}}\left(1 / L_{\varepsilon}(y)\right)$ in the case $\sigma \geqslant \sigma_{0}$.

Proof. This is Lemma 13 of Hildebrand and Tenenbaum [3]. For a more detailed proof, we refer the reader to the Exercise III.5.1 of [11].

Lemma 2.2. Let $k>0$ be fixed. We have

$$
\begin{array}{ll}
\alpha_{k} \leqslant 1+O\left(\frac{1}{\log y}\right) & \text { for } y \geqslant 2 \text { and } u \geqslant \varepsilon, \\
\alpha_{k}<1-\frac{\log 2}{\log y} & \text { for } y \geqslant y_{0}(\varepsilon, k) \text { and } u \geqslant k / \log 2+\varepsilon, \\
\alpha_{k} \geqslant \varepsilon / 2 & \text { for } y \geqslant y_{0}(\varepsilon, k) \text { and } \varepsilon \leqslant u \leqslant y^{1-\varepsilon}, \\
\alpha_{k} \asymp \frac{y}{u(\log y)^{2}} & \text { for } y \geqslant 2 \text { and } u \geqslant \frac{y}{\log y}, \\
\alpha_{k} \geqslant \frac{1}{11 \log y} & \text { for } y \geqslant 2 \text { and } \varepsilon \leqslant u \leqslant \frac{y}{\log y}, \\
\frac{y^{1-\alpha_{k}}-1}{\left(1-\alpha_{k}\right) \log y} \asymp \bar{u} & \text { for } y \geqslant 2 \text { and } u \geqslant \varepsilon,
\end{array}
$$


where $y_{0}(\varepsilon, k)$ is a sufficiently large constant depending on $\varepsilon, k$ only, and the expression on the left-hand side of (2.8) is to be interpreted as 1 if $\alpha_{k}=1$.

Proof. By (2.1) in Lemma 2.1, we have

$$
-\phi_{1}(1+c / \log y, y)=\left\{1+O\left(\frac{1}{L_{\varepsilon}(y)}\right)\right\} \frac{k\left(1-e^{-c}\right)}{c} \log y+O(1) \leqslant u \log y
$$

for $y \geqslant 2$ and $u \geqslant \varepsilon$ with a sufficiently large constant $c=c(\varepsilon, k)$; and

$$
-\phi_{1}(1-\log 2 / \log y, y)=\left\{1+O\left(\frac{1}{L_{\varepsilon}(y)}\right)\right\} \frac{k \log y}{\log 2}+O(1)<u \log y
$$

for $y \geqslant y_{0}(\varepsilon, k)$ and $u \geqslant k / \log 2+\varepsilon ;$ and

$$
-\phi_{1}(\varepsilon / 2, y)=\left\{1+O\left(\frac{1}{L_{\varepsilon}(y)}\right)\right\} \frac{k\left(y^{1-\varepsilon / 2}-1\right)}{1-\varepsilon / 2}+O(1)>y^{1-\varepsilon} \log y \geqslant u \log y
$$

for $y \geqslant y_{0}(\varepsilon, k)$ and $\varepsilon \leqslant u \leqslant y^{1-\varepsilon}$. Since $-\phi_{1}(\sigma, y)$ is a decreasing function of $\sigma$, we immediately deduce (2.3), (2.4) and (2.5).

For $y \geqslant 2$ and $u \geqslant \varepsilon$, we have

$$
\begin{aligned}
& u \log y=\sum_{p \leqslant y} \frac{k \log p}{p^{\alpha_{k}}-1} \leqslant \sum_{p \leqslant y} \frac{k}{\alpha_{k}} \leqslant \frac{10 k y}{\alpha_{k} \log y} \\
& u \log y=\sum_{p \leqslant y} \frac{k \log p}{p^{\alpha_{k}}-1} \geqslant \frac{k}{y^{\alpha_{k}}-1} \sum_{p \leqslant y} \log p \geqslant \frac{k y}{5\left(y^{\alpha_{k}}-1\right)} .
\end{aligned}
$$

It is easy to see that (2.9) implies the upper bound of (2.6) and that (2.10) implies

$$
\alpha_{k} \geqslant \frac{\log (1+k y / 5 u \log y)}{\log y}
$$

from which we deduce the lower bound of (2.6) if $u \geqslant y / \log y$, and (2.7) if $\varepsilon \leqslant u \leqslant$ $y / \log y$.

Finally we prove (2.8). If $u \geqslant y / \log y$, the right-hand side of $(2.8)$ is $y / \log y$. By (2.6), we have $\alpha_{k} \ll 1 / \log y$ and easily see that the left-hand side of (2.8) is $\asymp y / \log y$. If $\varepsilon \leqslant u \leqslant y / \log y$, by $(2.7)$ we have $\alpha_{k} \gg 1 / \log y$. Thus (2.1) in Lemma 2.1 implies

$$
u \log y \asymp \int_{1}^{y} \frac{d t}{t^{\alpha_{k}}}+1 \asymp \int_{1}^{y} \frac{d t}{t^{\alpha_{k}}}=\frac{y^{1-\alpha_{k}}-1}{1-\alpha_{k}} .
$$

This completes the proof. 
Lemma 2.3. For $y \geqslant 2, u \geqslant \varepsilon$ and any fixed positive integer $l$, we have

$$
0<(-1)^{l} \phi_{l}\left(\alpha_{k}, y\right) \asymp_{l}(u \log y)^{l} \bar{u}^{1-l} .
$$

Proof. Let $f(t):=1 /\left(e^{t}-1\right)=\sum_{n=1}^{\infty} e^{-n t}$, then

$$
(-1)^{i} f^{(i)}(t)=\sum_{n=1}^{\infty} n^{i} e^{-n t} \asymp_{i} e^{-t} \sum_{n=0}^{\infty}\left(\begin{array}{c}
n+i \\
i
\end{array}\right) e^{-n t}=\frac{e^{-t}}{\left(1-e^{-t}\right)^{i+1}} .
$$

Thus $(-1)^{l} \phi_{l}\left(\alpha_{k}, y\right)>0$ and

$$
(-1)^{l} \phi_{l}\left(\alpha_{k}, y\right)=(-1)^{l-1} k \sum_{p \leqslant y} f^{(l-1)}\left(\alpha_{k} \log p\right)(\log p)^{l} \asymp_{l} \sum_{p \leqslant y} \frac{(\log p)^{l} p^{-\alpha_{k}}}{\left(1-p^{-\alpha_{k}}\right)^{l}}
$$

If $u \geqslant y / \log y$, by $(2.6)$ we have $\alpha_{k} \ll 1 / \log y$. Thus we deduce

$$
(-1)^{l} \phi_{l}\left(\alpha_{k}, y\right) \asymp_{l} \sum_{p \leqslant y} \frac{(\log p)^{l}}{\left(\alpha_{k} \log p\right)^{l}} \asymp_{l} \frac{y}{\alpha_{k}^{l} \log y} \asymp_{l}(u \log y)^{l} \bar{u}^{1-l} .
$$

If $\varepsilon \leqslant u \leqslant y / \log y$, by (2.7) we have $\alpha_{k} \gg 1 / \log y$. Thus from (2.1) in Lemma 2.1 , we deduce that the last sum on the right-hand side of (2.11) is

$$
\begin{aligned}
& =\sum_{p \leqslant y} \frac{\log p}{p^{\alpha_{k}}-1}\left(\log p+\frac{\log p}{p^{\alpha_{k}}-1}\right)^{l-1} \leqslant \sum_{p \leqslant y} \frac{\log p}{p^{\alpha_{k}}-1}\left(\log p+\frac{1}{\alpha_{k}}\right)^{l-1} \\
& \ll(\log y)^{l-1} \sum_{p \leqslant y} \frac{\log p}{p^{\alpha_{k}}-1} \ll(\log y)^{l-1}\left(\int_{1}^{y} \frac{d t}{t^{\alpha_{k}}}+1\right) \\
& \asymp(\log y)^{l-1} \frac{y^{1-\alpha_{k}}-1}{1-\alpha_{k}} \asymp_{l}(u \log y)^{l} \bar{u}^{1-l} .
\end{aligned}
$$

In addition the last sum on the right-hand side of (2.11) is

$$
\begin{aligned}
& \geqslant \sum_{p \leqslant y} \frac{(\log p)^{l}}{p^{\alpha_{k}}} \gg \int_{y^{1 / 2}}^{y} \frac{(\log t)^{l-1}}{t^{\alpha_{k}}} d t \asymp(\log y)^{l-1} \int_{y^{1 / 2}}^{y} \frac{d t}{t^{\alpha_{k}}} \\
& \asymp(\log y)^{l-1} \int_{1}^{y} \frac{d t}{t^{\alpha_{k}}} \asymp(\log y)^{l-1} \frac{y^{1-\alpha_{k}}-1}{1-\alpha_{k}} \asymp_{l}(u \log y)^{l} \bar{u}^{1-l} .
\end{aligned}
$$

where we have used (2.8) and the inequality

$$
\int_{1}^{y^{1 / 2}} \frac{d t}{t^{\alpha_{k}}}=y^{\left(\alpha_{k}-1\right) / 2} \int_{y^{1 / 2}}^{y} \frac{d t}{t^{\alpha_{k}}} \ll \int_{y^{1 / 2}}^{y} \frac{d t}{t^{\alpha_{k}}} .
$$

This completes the proof. 
LEMMA 2.4. Let $k>0$ be fixed.

(i) For $x \geqslant 5$ and $2 \leqslant y \leqslant(\log x)^{2}$, we have

$$
\alpha_{k}(x, y)=\frac{\log (1+y / \log x)}{\log y}\left\{1+O\left(\frac{1}{\log y}\right)\right\} .
$$

(ii) For $x \geqslant 2$ and $(\log x)^{1+\varepsilon} \leqslant y \leqslant x$, we have

$$
\alpha_{k}(x, y)=1-\frac{\xi(u / k)}{\log y}+O\left(\frac{1}{L_{\varepsilon}(y) \log y}+\frac{1}{u(\log y)^{2}}\right) .
$$

Proof. Clearly the results desired are trivial if $y$ is bounded. Next we suppose $y \geqslant y_{0}(\varepsilon, k)$. We introduce the function $\alpha_{k, u}:=\alpha_{k}\left(y^{u}, y\right)$ and, define $v, w$ by

$$
\alpha_{k, v}:=\frac{\log (1+y / \log x)}{\log y}, \quad \alpha_{k, w}:=1-\frac{\xi}{\log y} .
$$

It is easy to verify $0<\alpha_{k, v}<\frac{3}{4}$ for $y \leqslant(\log x)^{2}, \alpha_{k, w} \asymp 1$ for $y \geqslant(\log x)^{1+\varepsilon}$ and

$$
\begin{aligned}
& \frac{y^{1-\alpha_{k, v}}-1}{\left(1-y^{-\alpha_{k, v}}\right)\left(1-\alpha_{k, v}\right)}=\frac{y\left\{1+O\left(y^{-1 / 4}\right)\right\}}{\left(y^{\alpha_{k, v}}-1\right)\left(1-\alpha_{k, v}\right)}=\frac{u \log y}{1-\alpha_{k, v}}\left\{1+O\left(y^{-1 / 4}\right)\right\}, \\
& \frac{y^{1-\alpha_{k, w}}-1}{\left(1-y^{-\alpha_{k, w}}\right)\left(1-\alpha_{k, w}\right)}=\frac{y^{1-\alpha_{k, w}}-1}{1-\alpha_{k, w}}\left\{1+O\left(\frac{1}{y^{\varepsilon}}\right)\right\}=u \log y\left\{1+O\left(y^{-\varepsilon}\right)\right\} .
\end{aligned}
$$

Thus (2.1) in Lemma 2.1 allows us to write

$$
\begin{aligned}
& v \log y=\left\{1+O\left(\frac{1}{\log y}\right)\right\} \frac{u \log y}{1-\alpha_{k, v}}+O(1), \\
& w \log y=\left\{1+O\left(\frac{1}{L_{\varepsilon}(y)}\right)\right\} u \log y+O(1),
\end{aligned}
$$

which imply

$$
\begin{aligned}
v \asymp u, & & |v-u| \asymp u\left(\alpha_{k, v}+1 / \log y\right), \\
w \asymp u, & & |w-u| \asymp u / L_{\varepsilon}(y)+1 / \log y .
\end{aligned}
$$

On differentiating $u \log y=-\phi_{1}\left(\alpha_{k, u}, y\right)$ with respect to $u$ and by using Lemma 2.3 , we get

$$
\alpha_{k, u}^{\prime}=-\frac{\log y}{\phi_{2}\left(\alpha_{k, u}, y\right)} \asymp \frac{1}{u \log y} \quad(u \geqslant \varepsilon) .
$$

From (2.12)-(2.14), we immediately deduce, for some $\eta_{1} \in(v, u)$ and some $\eta_{2} \in$ $(w, u)$,

$$
\begin{aligned}
&\left|\alpha_{k, u}-\alpha_{k, v}\right| \asymp\left|\alpha_{k, \eta_{1}}^{\prime}\right||v-u| \ll \frac{\bar{u}}{u \log y}\left(\alpha_{k, v}+\frac{1}{\log y}\right) \asymp \frac{\alpha_{k, v}}{\log y}, \\
&\left|\alpha_{k, u}-\alpha_{k, w}\right| \asymp\left|\alpha_{k, \eta_{2}}^{\prime}\right||w-u| \ll \frac{\bar{u}}{u \log y}\left(\frac{1}{L_{\varepsilon}(y)}+\frac{1}{u \log y}\right) \asymp \frac{1}{L_{\varepsilon}(y) \log y}+\frac{1}{u(\log y)^{2}} .
\end{aligned}
$$

This completes the proof. 
Lemma 2.5. Let $y \geqslant 2,0<\beta<1,|\tau| \leqslant Y_{\varepsilon}(y), s=1-\beta+i \tau$ and $\delta:=$ $\tau \log y-\operatorname{Arctg}(\tau / \beta)$. Let $\Lambda(n)$ be the von Mangoldt function. Then we have

$$
\sum_{n \leqslant y} \frac{\Lambda(n)\{1-\cos (\tau \log n)\}}{n^{1-\beta}}=\frac{y^{\beta}}{\beta}\left\{1-\frac{\beta \cos \delta}{\sqrt{\beta^{2}+\tau^{2}}}+O\left(y^{-\beta}+e^{-(\log y)^{\varepsilon / 2}}\right)\right\} .
$$

This is Corollary of Hildebrand and Tenenbaum [3, page 274].

LEMMA 2.6. For $y \geqslant y_{0}(\varepsilon, k), u \geqslant k / \log 2+\varepsilon$ and $s=\alpha_{k}+i \tau$ with $1 / \log y \leqslant$ $|\tau| \leqslant Y_{\varepsilon}(y)^{2}$, we have

$$
\left|\frac{\zeta(s, y)}{\zeta\left(\alpha_{k}, y\right)}\right|^{k} \ll \begin{cases}\exp \left\{-\frac{c_{2} \bar{u} \tau^{2}}{\left(1-\alpha_{k}\right)^{2}+\tau^{2}}\right\}, & \text { if } 1 / \log y \leqslant|\tau| \leqslant Y_{\varepsilon}(y)^{2}, \\ \exp \left\{-\frac{c_{3} y}{\log y} \log \left(1+\frac{\tau^{2} \phi_{2}\left(\alpha_{k}, y\right)}{(y / \log y)}\right)\right\} & \text { if }|\tau| \leqslant 1 / \log y .\end{cases}
$$

Proof. A simple calculation shows

$$
\left|\frac{1-p^{-\alpha_{k}}}{1-p^{-s}}\right|=\left(1+\frac{2(1-\cos (\tau \log p))}{p^{\alpha_{k}}\left(1-p^{-\alpha_{k}}\right)^{2}}\right)^{-1 / 2} .
$$

Using the inequality $\left\{1+2 v / t(1-1 / t)^{2}\right\}^{-1 / 2} \leqslant e^{-v / t}(0 \leqslant 2 v \leqslant 1<t)$ with $v=1-\cos (\tau \log p)$ and $t=p^{\alpha_{k}}$, we find

$$
|\zeta(s, y)|^{k} \leqslant \zeta\left(\alpha_{k}, y\right)^{k} e^{-k U},
$$

where

$$
U:=\sum_{p \leqslant y} \frac{1-\cos (\tau \log p)}{p^{\alpha_{k}}} .
$$

We need to study the lower bound for $U$. For this we write

$$
V:=\frac{1}{\log y} \sum_{n \leqslant y} \frac{\Lambda(n)\{1-\cos (\tau \log n)\}}{n^{\alpha_{k}}} \leqslant U+2 W,
$$

where

$$
W:=\frac{1}{\log y} \sum_{\nu \geqslant 2} \sum_{p^{\nu} \leqslant y} \frac{\log p}{p^{\alpha_{k} \nu}} \leqslant \sum_{p \leqslant \sqrt{y}} \frac{1}{p^{2 \alpha_{k}}} \ll y^{1 / 2-\alpha_{k}}+1 .
$$

By (2.4), Lemma 2.5 is applicable with $\beta=1-\alpha_{k}$ and with $\varepsilon / 2$ in place of $\varepsilon$. Thus we find

$$
V=\frac{y^{1-\alpha_{k}}}{\left(1-\alpha_{k}\right) \log y}\left\{1-\frac{\left(1-\alpha_{k}\right) \cos \delta}{\sqrt{\left(1-\alpha_{k}\right)^{2}+\tau^{2}}}+O\left(e^{-(\log y)^{\varepsilon / 4}}+y^{\alpha_{k}-1}\right)\right\}
$$


where $\delta:=\tau \log y-\operatorname{Arctg}\left\{\tau /\left(1-\alpha_{k}\right)\right\}$.

Firstly we consider the case $1 / \log y \leqslant|\tau| \leqslant Y_{\varepsilon}^{2}(y)$. We need to prove

$$
U \gg \frac{\bar{u} \tau^{2}}{\left(1-\alpha_{k}\right)^{2}+\tau^{2}}
$$

If $u \geqslant y / \log y,(2.6)$ implies $\alpha_{k} \ll 1 / \log y$. Thus from (2.17)-(2.19), we deduce

$$
\begin{aligned}
U & \gg \frac{y}{\log y}\left\{1-\frac{1-\alpha_{k}}{\sqrt{\left(1-\alpha_{k}\right)^{2}+\tau^{2}}}+O\left(e^{-(\log y)^{\varepsilon / 4}}\right)\right\} \\
& \gg \frac{y}{\log y}\left\{\frac{\tau^{2}}{\left(1-\alpha_{k}\right)^{2}+\tau^{2}}+O\left(e^{-(\log y)^{\varepsilon / 4}}\right)\right\},
\end{aligned}
$$

which implies (2.20).

When $k / \log 2+\varepsilon \leqslant u \leqslant y / \log y$, by (2.17)-(2.19) and (2.8), we have

$$
\begin{aligned}
U & \geqslant \frac{y^{1-\alpha_{k}}}{\left(1-\alpha_{k}\right) \log y}\left\{1-\frac{\left(1-\alpha_{k}\right) \cos \delta}{\sqrt{\left(1-\alpha_{k}\right)^{2}+\tau^{2}}}+O\left(e^{-(\log y)^{\varepsilon / 4}}+\frac{\left|1-\alpha_{k}\right| \log y}{y^{1-\alpha_{k}}}\right)\right\} \\
(2.21) & \geqslant \frac{y^{1-\alpha_{k}}-1}{\left(1-\alpha_{k}\right) \log y}\left\{1-\frac{1-\alpha_{k}}{\sqrt{\left(1-\alpha_{k}\right)^{2}+\tau^{2}}}+O\left(e^{-(\log y)^{\varepsilon / 4}}+\frac{\left|1-\alpha_{k}\right| \log y}{y^{1-\alpha_{k}}-1}\right)\right\} \\
& \gg u\left\{\frac{\tau^{2}}{\left(1-\alpha_{k}\right)^{2}+\tau^{2}}+O\left(e^{-(\log y)^{\varepsilon / 4}}+\frac{1}{u}\right)\right\} .
\end{aligned}
$$

Since $|\tau| \geqslant 1 / \log y$, we have $\left(\tau /\left(1-\alpha_{k}\right)\right)^{2} \geqslant(\log y)^{-2}$. If $1 \leqslant u \leqslant y^{1-\varepsilon}$, Lemma 2.4 implies $\left(1-\alpha_{k}\right) \log y \ll \log (2 u)$. Thus $\left(\tau /\left(1-\alpha_{k}\right)\right)^{2} \geqslant\left\{\left(1-\alpha_{k}\right) \log y\right\}^{-2} \gg$ $\{\log (2 u)\}^{-2}$. When $y^{1-\varepsilon} \leqslant u \leqslant y / \log y$, we have $\left(\tau /\left(1-\alpha_{k}\right)\right)^{2} \geqslant(\log y)^{-2} \gg$ $\{\log (2 u)\}^{-2}$. Therefore we have

$$
\left\{\tau /\left(1-\alpha_{k}\right)\right\}^{2} \gg \max \left\{(\log y)^{-2},(\log 2 u)^{-2}\right\},
$$

which implies

$$
\frac{\tau^{2}}{\left(1-\alpha_{k}\right)^{2}+\tau^{2}} \geqslant \frac{1}{2} \min \left\{1,\left(\frac{\tau}{1-\alpha_{k}}\right)^{2}\right\} \gg \max \left\{\frac{1}{(\log y)^{2}}, \frac{1}{(\log 2 u)^{2}}\right\} .
$$

Hence the error term in (2.21) can be absorbed by the main term and we get (2.20).

Secondly we consider the case $|\tau| \leqslant 1 / \log y$.

The following inequalities are easy to verify:

$$
\begin{aligned}
& 2 t^{2} / \pi^{2} \leqslant 1-\cos t \leqslant t^{2} / 2 \quad(|t| \leqslant \pi) \\
& \sigma \log t \leqslant t^{\sigma}-1 \leqslant \sigma t^{\sigma} \log t \quad(t \geqslant 2, \sigma>0) \\
& \log \left(1+\left(4 / \pi^{2}\right) t\right) \geqslant\left(4 / \pi^{2}\right) \log (1+t) \quad(t \geqslant 0) \\
& e^{t}\left(1-e^{-t}\right)^{2} \geqslant t^{2} \quad(t \in \mathbb{R})
\end{aligned}
$$


From (2.15) and (2.22)-(2.24), we deduce, for $p \leqslant y$,

$$
\begin{aligned}
\left|\frac{1-p^{-\alpha_{k}}}{1-p^{-s}}\right| & \leqslant \exp \left\{-\frac{1}{2} \log \left(1+\frac{4 \tau^{2} \log ^{2} p}{\pi^{2}\left(1-p^{-\alpha_{k}}\right)^{2} p^{\alpha_{k}}}\right)\right\} \\
& \leqslant \exp \left\{-\frac{1}{2} \log \left(1+\frac{4 \tau^{2}}{\pi^{2} \alpha_{k}^{2} y^{\alpha_{k}}}\right)\right\} \leqslant \exp \left\{-\frac{2}{\pi^{2}} \log \left(1+\frac{\tau^{2}}{\alpha_{k}^{2} y^{\alpha_{k}}}\right)\right\} .
\end{aligned}
$$

If $u \geqslant y / \log y$, then (2.6) and Lemma 2.3 imply

$$
\frac{1}{\alpha_{k}^{2} y^{\alpha_{k}}} \asymp \frac{1}{\alpha_{k}^{2}} \asymp \frac{u^{2}(\log y)^{4}}{y^{2}} \asymp \frac{\phi_{2}\left(\alpha_{k}, y\right)}{(y / \log y)} .
$$

Taking the product over $p \leqslant y$ yields the second desired inequality for $u \geqslant y / \log y$.

If $u \leqslant y / \log y$, then (2.22), (2.25) and (2.7) yield, for $|\tau| \leqslant 1 / \log y$,

$$
\frac{2(1-\cos (\tau \log p))}{\left(1-p^{-\alpha_{k}}\right)^{2} p^{\alpha_{k}}} \leqslant\left(\frac{\tau \log p}{\alpha_{k} \log p}\right)^{2}=\left(\frac{\tau}{\alpha_{k}}\right)^{2} \leqslant c_{4},
$$

where $c_{4}=c_{4}(k)$ is sufficiently large constant. By (2.15), (2.22), and the inequality $(1+t)^{-1 / 2} \leqslant e^{-t / 2\left(1+c_{4}\right)}\left(0 \leqslant t \leqslant c_{4}\right)$ we deduce, $c_{5}:=2 / \pi^{2}\left(1+c_{4}\right)$,

$$
\left|\frac{1-p^{-\alpha_{k}}}{1-p^{-s}}\right| \leqslant \exp \left\{-\frac{1-\cos (\tau \log p)}{\left(1+c_{4}\right)\left(1-p^{-\alpha_{k}}\right)^{2} p^{\alpha_{k}}}\right\} \leqslant \exp \left\{-\frac{c_{5} \tau^{2}(\log p)^{2}}{\left(1-p^{-\alpha_{k}}\right)^{2} p^{\alpha_{k}}}\right\}
$$

and $|\zeta(s, y)|^{k} \leqslant \zeta\left(\alpha_{k}, y\right)^{k} e^{-c_{5} \tau^{2} \phi_{2}\left(\alpha_{k}, y\right)}$. Since $\frac{\tau^{2} \phi_{2}\left(\alpha_{k}, y\right)}{(y / \log y)} \asymp \frac{\tau^{2} u(\log y)^{3}}{y} \leqslant 1$, the
preceding inequality implies the desired result.

Lemma 2.7. For $y \geqslant y_{0}(\varepsilon, k), u \geqslant k / \log 2+\varepsilon$ and $1 \leqslant z \leqslant Y_{\varepsilon}(y)$, we have

$$
\Delta_{k}(x, y, z):=\sum_{\substack{x<n \leqslant x+x / z \\ P(n) \leqslant y}} \tau_{k}(n) \ll x^{\alpha_{k}} \zeta\left(\alpha_{k}, y\right)^{k}\left(1 / z+e^{-c_{6} \bar{u}}\right) .
$$

Proof. Noticing that $\tau_{k}(n)>0$ and $1 \leqslant e^{\left\{1-z^{2} \log ^{2}(x / n)\right\} / 2}$ for $x<n \leqslant x+x / z$, we have

$$
\Delta_{k}(x, y, z) \ll \sum_{P(n) \leqslant y} \tau_{k}(n) e^{-\{z \log (x / n)\}^{2} / 2} .
$$

By the Laplace inversion formula, we easily see, for $\sigma, v \in \mathbb{R}$,

$$
e^{-v^{2} / 2}=\frac{1}{i \sqrt{2 \pi}} \int_{\sigma-i \infty}^{\sigma+i \infty} e^{s^{2} / 2-v s} d s=\frac{e^{\sigma^{2} / 2-\sigma v}}{\sqrt{2 \pi}} \int_{-\infty}^{+\infty} e^{-\tau^{2} / 2+i \tau(\sigma-v)} d \tau .
$$

Using this relation with $v=-z \log (x / n)$ and $\sigma=\alpha_{k} / z$, it follows that 


$$
\begin{aligned}
\Delta_{k}(x, y, z) & \ll e^{\alpha_{k}^{2} / 2 z^{2}} \sum_{P(n) \leqslant y} \tau_{k}(n) \int_{-\infty}^{+\infty} e^{i \alpha_{k} \tau / z-\tau^{2} / 2}\left(\frac{x}{n}\right)^{\alpha_{k}+i z \tau} d \tau \\
& \ll \int_{-\infty}^{+\infty} e^{i \alpha_{k} \tau / z-\tau^{2} / 2} x^{\alpha_{k}+i z \tau} \zeta\left(\alpha_{k}+i z \tau, y\right)^{k} d \tau \\
& \ll \frac{x^{\alpha_{k}}}{z} \int_{-\infty}^{+\infty} e^{-\tau^{2} / 2 z^{2}}\left|\zeta\left(\alpha_{k}+i \tau, y\right)\right|^{k} d \tau .
\end{aligned}
$$

In order to bound the last integral, we split the interval of integration into three parts: $|\tau| \leqslant 1,1<|\tau| \leqslant z^{2}$ or $|\tau| \geqslant z^{2}$, and use $I_{1}, I_{2}, I_{3}$ to denote the corresponding contributions. Clearly $I_{1} \ll \zeta\left(\alpha_{k}, y\right)^{k}$ and $I_{3} \ll \zeta\left(\alpha_{k}, y\right)^{k}$. In addition the first inequality in Lemma 2.6 implies

$$
I_{2} \ll \zeta\left(\alpha_{k}, y\right)^{k} e^{-c_{6} \bar{u}} \int_{1}^{z^{2}} e^{-\tau^{2} / 2 z^{2}} d \tau \ll z \zeta\left(\alpha_{k}, y\right)^{k} e^{-c_{6} \bar{u}} .
$$

Inserting these estimations into (2.26), we obtain the required result.

\section{Proof of Theorem 2}

Since $\xi(u / k)=\log u+O(\log \log u)$, we easily see that Lemma 2.4 implies (1.6). Now we prove (1.7). In view of Lemma 2.3 , we can suppose $y \geqslant y_{0}(\varepsilon, k)$ and $u \geqslant u_{0}(\varepsilon, k)$. By integration by parts and by $(2.8)$, it follows

$$
\begin{aligned}
\int_{1}^{y} \frac{\log t}{t^{\alpha_{k}}} d t & =\log y \frac{y^{1-\alpha_{k}}-1}{1-\alpha_{k}}+\frac{\log y}{1-\alpha_{k}}-\frac{y^{1-\alpha_{k}}-1}{\left(1-\alpha_{k}\right)^{2}} \\
& =\log y \frac{y^{1-\alpha_{k}}-1}{1-\alpha_{k}}\left\{1+O\left(\frac{1}{\left(1-\alpha_{k}\right) \log y}\right)\right\} \\
& =\log y \frac{y^{1-\alpha_{k}}-1}{1-\alpha_{k}}\left\{1+O\left(\frac{1}{\log (2 u)}+\frac{1}{\log y}\right)\right\} .
\end{aligned}
$$

where we have used Lemma 2.4 in the last estimate. Thus Lemma 2.1 allows us to deduce

$$
\begin{aligned}
\phi_{2}\left(\alpha_{k}, y\right) & =\left\{1+O\left(\frac{1}{\log (2 u)}+\frac{1}{\log y}\right)\right\} \frac{\log y}{\left(1-y^{-\alpha_{k}}\right)^{2}} \frac{y^{1-\alpha_{k}}-1}{1-\alpha_{k}}+O(1) \\
& =\left\{1+O\left(\frac{1}{\log (2 u)}+\frac{1}{\log y}\right)\right\} \frac{\log y}{1-y^{-\alpha_{k}}}\left\{-\phi_{1}\left(\alpha_{k}, y\right)+O(1)\right\}+O(1) \\
& =\left\{1+O\left(\frac{1}{\log (2 u)}+\frac{1}{\log y}\right)\right\} \frac{(\log x)(\log y)}{1-y^{-\alpha_{k}}}+O(1) .
\end{aligned}
$$

If $y \geqslant u(\log y)^{2}$, then by using (1.6) we easily see that $y^{-\alpha_{k}} \ll 1 / \log y$. Thus

$$
\frac{1}{1-y^{-\alpha_{k}}}=\left(1+\frac{\log x}{y}\right)\left\{1+O\left(\frac{1}{\log y}\right)\right\} .
$$


If $y \leqslant u(\log y)^{2}$, then Lemma 2.4(i) implies

$$
y^{-\alpha_{k}}=\left(1+\frac{y}{\log x}\right)^{-1+O(1 / \log y)}=\left(1+\frac{y}{\log x}\right)^{-1}\left\{1+O\left(\frac{y}{u(\log y)^{2}}\right)\right\},
$$

from which we easily see that (3.3) also holds in this circumstance. Now inserting (3.3) into (3.2) yields the desired estimation (1.7).

Finally we prove (ii) of Theorem 2. By using Lemma 2.4(ii), we have

$$
x^{\alpha_{k}}=x e^{-u \xi+O\left(u / L_{\varepsilon}(y)+1 / \log y\right)}, \quad \frac{1}{\alpha_{k}}=1+O\left(\frac{\log (2 u)}{\log y}\right)=e^{O(\log (2 u) / \log y)} .
$$

In order to evaluate $\zeta\left(\alpha_{k}, y\right)^{k}$, we write

$$
\zeta\left(\alpha_{k}, y\right)^{k}=\zeta(1, y)^{k} \exp \left\{-\int_{\alpha_{k}}^{1} \phi_{1}(\sigma, y) d \sigma\right\} .
$$

The Mertens theorem implies

$$
\zeta(1, y)^{k}=(\log y)^{k} e^{k \gamma+O(1 / \log y)} .
$$

In view of (2.5), (2.1) in Lemma 2.1 allows us to deduce

$$
-\int_{\alpha_{k}}^{1} \phi_{1}(\sigma, y) d \sigma=\left\{1+O\left(\frac{1}{L_{\varepsilon}(y)}\right)\right\} k \int_{\alpha_{k}}^{1} \frac{y^{1-\sigma}-1}{1-\sigma} d \sigma+O\left(\left|1-\alpha_{k}\right|\right) .
$$

By change of variable $(1-\sigma) \log y=v$ and Lemma 2.4, we have

$$
\int_{\alpha_{k}}^{1} \frac{y^{1-\sigma}-1}{1-\sigma} d \sigma=\int_{0}^{\xi+O\left(1 / L_{\varepsilon}(y)+1 / u \log y\right)} \frac{e^{v}-1}{v} d v=I(\xi)+O\left(\frac{u}{L_{\varepsilon}(y)}+\frac{1}{\log y}\right),
$$

where we have used the estimate $I(\xi) \asymp u$. Inserting into (3.7) and using Lemma 2.4 yield

$$
-\int_{\alpha_{k}}^{1} \phi_{1}(\sigma, y) d \sigma=\sigma_{0}+O\left(\frac{u}{L_{\varepsilon}(y)}+\frac{1}{\log y}\right) .
$$

Combining (3.6) and (3.8) with (3.5) yields

$$
\zeta\left(\alpha_{k}, y\right)^{k}=(\log y)^{k} e^{k \gamma+\sigma_{0}+O\left(u / L_{\varepsilon}(y)+1 / \log y\right)} .
$$

Finally we evaluate $\phi_{2}\left(\alpha_{k}, y\right)$. Define $\alpha_{k, w}:=1-\xi / \log y$. From Lemma 2.4, we deduce

$$
\begin{aligned}
\left|\int_{1}^{y}\left(\frac{\log t}{t^{\alpha_{k}}}-\frac{\log t}{t^{\alpha_{k, w}}}\right) d t\right| & \leqslant \int_{1}^{y} \frac{\log t}{t^{\alpha_{k, w}}}\left|t^{\alpha_{k, w}-\alpha_{k}}-1\right| d t \ll\left|\alpha_{k, w}-\alpha_{k}\right| \log y \int_{1}^{y} \frac{\log t}{t^{\alpha_{k, w}}} d t \\
& \ll\left(\frac{1}{L_{\varepsilon}(y)}+\frac{1}{u \log y}\right) \int_{1}^{y} \frac{\log t}{t^{\alpha_{k, w}}} d t .
\end{aligned}
$$


In addition by the first estimate in (3.1), we have

$$
\int_{1}^{y} \frac{\log t}{t^{\alpha_{k, w}}} d t=\log y \frac{y^{1-\alpha_{k, w}}-1}{1-\alpha_{k, w}}+\frac{\log y}{1-\alpha_{k, w}}-\frac{y^{1-\alpha_{k, w}}-1}{\left(1-\alpha_{k, w}\right)^{2}}=\frac{\sigma_{2}(\log y)^{2}}{k}
$$

By using (2.2) and these estimations, we obtain

$$
\begin{aligned}
\phi_{2}\left(\alpha_{k}, y\right) & =\left\{k+O\left(\frac{1}{L_{\varepsilon}(y)}\right)\right\}\left\{\int_{1}^{y} \frac{\log t}{t^{\alpha_{k}, w}} d t+\int_{1}^{y}\left(\frac{\log t}{t^{\alpha_{k}}}-\frac{\log t}{t^{\alpha_{k}, w}}\right) d t\right\}+O(1) \\
& =\left\{1+O\left(\frac{1}{L_{\varepsilon}(y)}+\frac{1}{u \log y}\right)\right\} \sigma_{2}(\log y)^{2}=\sigma_{2}(\log y)^{2} e^{O\left(1 / L_{\varepsilon}(y)+1 / u \log y\right)},
\end{aligned}
$$

Now (1.8) follows from (3.4), (3.9) and (3.10); and (1.9) from (1.8) and Théorème 1 of $[7]$ :

$$
\rho_{k}(u)=\frac{e^{k \gamma+\sigma_{0}-u \xi}}{\sqrt{2 \pi \sigma_{2}}}\left\{1+O\left(\frac{1}{u}\right)\right\} .
$$

This completes the proof.

\section{Proof of Theorem 1}

By Theorem 2 and Smida's asymptotic formula (1.4), it is easy to see that Theorem 1 holds for $1 \leqslant u \leqslant(\log \log y)^{2}$. In addition (2.6) and Lemma 2.3 imply

$$
\alpha_{k} \sqrt{\phi_{2}\left(\alpha_{k}, y\right)} \ll \begin{cases}\sqrt{u} \log y, & \text { if } u \leqslant y / \log y, \\ \sqrt{y / \log y,} & \text { if } u \geqslant y / \log y\end{cases}
$$

Thus the conclusion of Theorem 1 is a simple consequence of Rankin's method if $y \leqslant y_{0}(\varepsilon, k)$. Next we shall prove Theorem 1 for the range $y \geqslant y_{0}(\varepsilon, k)$ and $u \geqslant(\log \log y)^{2}$ in two steps which we formulate as lemmas. For simplicity, we write $\lambda_{0}:=\phi\left(\alpha_{k}, y\right)$ and $\lambda_{l}:=\phi_{l}\left(\alpha_{k}, y\right)(l \geqslant 1)$.

LEMMA 4.1. For $y \geqslant y_{0}(\varepsilon, k)$ and $u \geqslant k / \log 2+\varepsilon$, we have

$$
S_{k}(x, y)=\frac{1}{2 \pi i} \int_{\alpha_{k}-i / \log y}^{\alpha_{k}+i / \log y} \zeta(s, y)^{k} \frac{x^{s}}{s} d s+O\left(x^{\alpha_{k}} \zeta\left(\alpha_{k}, y\right)^{k} R_{0}(x, y)\right)
$$

where $R_{0}(x, y):=Y_{\varepsilon}(y)^{-1}+e^{-c_{7} u /(\log 2 u)^{2}}$. In particular for $u \geqslant(\log \log y)^{2}$, we have

$$
R_{0}(x, y) \ll 1 /\left\{\alpha_{k} \sqrt{\lambda_{2}} \bar{u}\right\}
$$

Proof. Applying the Perron formula (cf. [10, Théorème II.2.2]), we have

$$
S_{k}(x, y)=\frac{1}{2 \pi i} \int_{\alpha_{k}-i T}^{\alpha_{k}+i T} \zeta(s, y)^{k} \frac{x^{s}}{s} d s+O\left(R_{1}\right)
$$


where $T:=R_{0}(x, y)^{-2}$ and

$$
R_{1}:=x^{\alpha_{k}} \sum_{P(n) \leqslant y} \frac{\tau_{k}(n)}{n^{\alpha_{k}}(1+T|\log (x / n)|)} .
$$

In order to bound $R_{1}$, we split the range of summation into two parts: $|\log (x / n)|>$ $1 / \sqrt{T}$ or $|\log (x / n)| \leqslant 1 / \sqrt{T}$, and easily see that

$$
R_{1} \leqslant \frac{x^{\alpha_{k}} \zeta\left(\alpha_{k}, y\right)^{k}}{\sqrt{T}}+x^{\alpha_{k}} \sum_{|\log (x / n)| \leqslant 1 / \sqrt{T}} \frac{\tau_{k}(n)}{n^{\alpha_{k}}} .
$$

Since $|\log (x / n)| \leqslant 1 / \sqrt{T} \Rightarrow|x-n| \leqslant c_{8} x / \sqrt{T}$, Lemma 2.7 shows that the second member on the right-hand side is

$$
\ll \sum_{\substack{|x-n| \leqslant c_{8} x / \sqrt{T} \\ P(n) \leqslant y}} \tau_{k}(n) \ll x^{\alpha_{k}} \zeta\left(\alpha_{k}, y\right)^{k}\left(\frac{1}{\sqrt{T}}+e^{-c_{9} \bar{u}}\right) \ll \frac{x^{\alpha_{k}} \zeta\left(\alpha_{k}, y\right)^{k}}{\sqrt{T}} .
$$

Consequently

$$
R_{1} \ll x^{\alpha_{k}} \zeta\left(\alpha_{k}, y\right)^{k} / \sqrt{T} .
$$

From the first inequality in Lemma 2.4, we deduce

$$
\int_{\substack{\sigma=\alpha_{k} \\ 1 / \log y \leqslant|\tau| \leqslant T}} \zeta(s, y)^{k} \frac{x^{s}}{s} d s \ll x^{\alpha_{k}} \zeta\left(\alpha_{k}, y\right)^{k} \int_{1 / \log y}^{T} \frac{e^{-c_{2} \bar{u} \tau^{2} /\left\{\left(1-\alpha_{k}\right)^{2}+\tau^{2}\right\}}}{\alpha_{k}+\tau} d \tau .
$$

Let $\eta_{k}:=\max \left\{\left|1-\alpha_{k}\right|, 1 / \log y\right\}$, then $\log \left\{\left(\alpha_{k}+\eta_{k}\right) /\left(\alpha_{k}+1 / \log y\right)\right\} \ll \log y$. In addition $(2.8)$ implies $\left(1-\alpha_{k}\right) \log y \ll \log (2 \bar{u})$. Thus the last integral on the right-hand side of (4.4) is,

$$
\begin{aligned}
& \ll \int_{1 / \log y}^{\eta_{k}} e^{-c_{2} \bar{u} \tau^{2} / 2\left(1-\alpha_{k}\right)^{2}} \frac{d \tau}{\alpha_{k}+\tau}+\int_{\eta_{k}}^{T} e^{-c_{2} \bar{u} / 2} \frac{d \tau}{\alpha_{k}+\tau} \\
& \ll e^{-c_{2} \bar{u} / 2\left(\left(1-\alpha_{k}\right) \log y\right)^{2}} \log \left(\frac{\alpha_{k}+\eta_{k}}{\alpha_{k}+1 / \log y}\right)+e^{-c_{2} \bar{u} / 2} \log T \\
& \ll e^{-c_{10} \bar{u} /(\log 2 \bar{u})^{2}} \log y+e^{-c_{2} \bar{u} / 2} \log T \ll e^{-c_{10} \bar{u} /(\log 2 \bar{u})^{2}} \log T \ll 1 / \sqrt{T} .
\end{aligned}
$$

Hence

$$
\int_{\substack{\sigma=\alpha_{k} \\ 1 / \log y \leqslant|\tau| \leqslant T}} \zeta(s, y)^{k} \frac{x^{s}}{s} d s \ll x^{\alpha_{k}} \zeta\left(\alpha_{k}, y\right)^{k} / \sqrt{T} .
$$

Now the desired result follows from (4.2), (4.3) and (4.5).

Finally by using (4.1), we easily verify $R_{0}(x, y) \ll 1 /\left\{\alpha_{k} \sqrt{\lambda_{2}} \bar{u}\right\}$ provided $u \geqslant$ $(\log \log y)^{2}$. This completes the proof. 
Lemma 4.2. For $y \geqslant y_{0}(\varepsilon, k)$ and $u \geqslant k / \log 2+\varepsilon$, we have

$$
\frac{1}{2 \pi i} \int_{\alpha_{k}-i / \log y}^{\alpha_{k}+i / \log y} \zeta(s, y)^{k} \frac{x^{s}}{s} d s=\frac{x^{\alpha_{k}} \zeta\left(\alpha_{k}, y\right)^{k}}{\alpha_{k} \sqrt{2 \pi \lambda_{2}}}\left\{1+O\left(\frac{1}{\bar{u}}\right)\right\}
$$

Further the same formula also holds for

$$
\frac{1}{2 \pi} \int_{\alpha_{k}-i / \log y}^{\alpha_{k}+i / \log y}\left|\zeta(s, y)^{k} \frac{x^{s}}{s}\right||d s|
$$

Proof. We first write, for $s=\alpha_{k}+i \tau$,

$$
\zeta(s, y)^{k} \frac{x^{s}}{s}=\frac{x^{\alpha_{k}}}{\alpha_{k}+i \tau} e^{\phi\left(\alpha_{k}+i \tau, y\right)+i \tau \log y} .
$$

Developing $\phi\left(\alpha_{k}+i \tau, y\right)$ at $\tau=0$, we have for $|\tau| \leqslant \delta:=\bar{u}^{2 / 3} /(u \log y)$

$$
\phi\left(\alpha_{k}+i \tau, y\right)=\lambda_{0}+i \lambda_{1} \tau-\frac{\lambda_{2}}{2} \tau^{2}-\frac{i \lambda_{3}}{6} \tau^{3}+O\left(\lambda_{4} \tau^{4}\right)
$$

where we have used the trivial estimation $\left|\phi_{4}\left(\alpha_{k}+i \tau, y\right)\right| \leqslant \phi_{4}\left(\alpha_{k}, y\right)=\lambda_{4}$ for $\tau \in \mathbb{R}$. Since $|\tau| \leqslant \delta$, Lemma 2.3 implies that $\lambda_{3} \tau^{3}$ and $\lambda_{4} \tau^{4}$ and, (2.6) and (2.7) imply $\tau / \alpha_{k} \ll \bar{u}^{-1 / 3}$. Thus we can write, for $|\tau| \leqslant \delta$,

$$
\begin{aligned}
e^{-i \lambda_{3} \tau^{3} / 6+O\left(\lambda_{4} \tau^{4}\right)} & =1-\frac{i \lambda_{3}}{6} \tau^{3}+O\left(\lambda_{3}^{2} \tau^{6}+\lambda_{4} \tau^{4}\right), \\
\frac{1}{\alpha_{k}+i \tau} & =\frac{1}{\alpha_{k}}\left\{1-\frac{i}{\alpha_{k}} \tau+O\left(\frac{\tau^{2}}{\alpha_{k}^{2}}\right)\right\} .
\end{aligned}
$$

Inserting these into (4.6) and noticing that $\lambda_{1}+\log x=0$ yield

$\zeta(s, y)^{k} \frac{x^{s}}{s}=\frac{x^{\alpha_{k}} \zeta\left(\alpha_{k}, y\right)^{k}}{\alpha_{k}} e^{-\lambda_{2} \tau^{2} / 2}\left\{1-\frac{i}{\alpha_{k}} \tau-\frac{i \lambda_{3}}{6} \tau^{3}+O\left(\lambda_{3}^{2} \tau^{6}+\lambda_{4} \tau^{4}+\alpha_{k}^{-2} \tau^{2}\right)\right\}$,

from which

$$
\begin{aligned}
\frac{1}{2 \pi i} \int_{\alpha_{k}-i \delta}^{\alpha_{k}+i \delta} \zeta(s, y)^{k} \frac{x^{s}}{s} d s & \\
& =\frac{x^{\alpha_{k}} \zeta\left(\alpha_{k}, y\right)^{k}}{2 \pi \alpha_{k}} \int_{-\delta}^{\delta} e^{-\lambda_{2} \tau^{2} / 2}\left\{1+O\left(\lambda_{3}^{2} \tau^{6}+\lambda_{4} \tau^{4}+\alpha_{k}^{-2} \tau^{2}\right)\right\} d \tau .
\end{aligned}
$$

A simple calculation shows

$$
\int_{-\delta}^{\delta} e^{-\lambda_{2} \tau^{2} / 2} d \tau=\sqrt{\frac{2 \pi}{\lambda_{2}}}\left\{1+O\left(e^{-\lambda_{2} \delta^{2} / 2}\right)\right\}=\sqrt{\frac{2 \pi}{\lambda_{2}}}\left\{1+O\left(e^{-c_{11} \bar{u}^{1 / 3}}\right)\right\}
$$


and

$$
\int_{-\delta}^{\delta} e^{-\lambda_{2} \tau^{2} / 2}\left(\lambda_{3}^{2} \tau^{6}+\lambda_{4} \tau^{4}+\alpha_{k}^{-2} \tau^{2}\right) d \tau \ll \frac{1}{\sqrt{\lambda_{2}}}\left(\lambda_{3}^{2} \lambda_{2}^{-3}+\lambda_{4} \lambda_{2}^{-2}+\alpha_{k}^{-2} \lambda_{2}^{-1}\right) \ll \frac{1}{\sqrt{\lambda_{2}} \bar{u}} .
$$

This proves

$$
\frac{1}{2 \pi i} \int_{\alpha_{k}-i \delta}^{\alpha_{k}+i \delta} \zeta(s, y)^{k} \frac{x^{s}}{s} d s=\frac{x^{\alpha_{k}} \zeta\left(\alpha_{k}, y\right)^{k}}{\alpha_{k} \sqrt{2 \pi \lambda_{2}}}\left\{1+O\left(\frac{1}{\bar{u}}\right)\right\}
$$

It remains to verify

$$
\int_{\substack{\sigma=\alpha_{k} \\ \delta \leqslant|\tau| \leqslant 1 / \log y}} \zeta(s, y)^{k} \frac{x^{s}}{s} d s \ll \frac{x^{\alpha_{k}} \zeta\left(\alpha_{k}, y\right)^{k}}{\alpha_{k} \sqrt{\lambda_{2}}} \frac{1}{\bar{u}}
$$

By using the second inequality in Lemma 2.6, the left-hand side is

$$
\begin{aligned}
& \ll \int_{\delta}^{1 / \log y} \frac{x^{\alpha_{k}} \zeta\left(\alpha_{k}, y\right)^{k}}{\alpha_{k}+\tau}\left(1+\frac{\lambda_{2} \tau^{2} \log y}{y}\right)^{-c_{3} y / \log y} d \tau \\
& \ll \frac{x^{\alpha_{k}} \zeta\left(\alpha_{k}, y\right)^{k}}{\alpha_{k} \sqrt{\lambda_{2}}} \int_{\delta \sqrt{\lambda_{2}}}^{+\infty}\left(1+\frac{\tau^{2} \log y}{y}\right)^{-c_{3} y / \log y} d \tau .
\end{aligned}
$$

In order to bound the last integral, we split $\left[\delta \sqrt{\lambda_{2}}, \infty\right)$ into two parts: $\left[\delta \sqrt{\lambda_{2}}\right.$, $\sqrt{y / \log y}]$ and $[\sqrt{y / \log y}, \infty)$, and use $I_{1}, I_{2}$ to denote the corresponding contributions. Clearly we have

$$
\begin{aligned}
& I_{1} \ll \int_{\delta \sqrt{\lambda_{2}}}^{\sqrt{y / \log y}} e^{-c_{3} \tau^{2} / 2} d \tau \ll \frac{e^{-c_{3} \delta^{2} \lambda_{2}}}{\delta \sqrt{\lambda_{2}}} \asymp \frac{e^{-c_{12} \bar{u}^{1 / 3}}}{\bar{u}^{1 / 3}} \ll \frac{1}{\bar{u}}, \\
& I_{2} \ll \int_{\sqrt{y / \log y}}^{\infty}\left(\frac{2 \tau^{2} \log y}{y}\right)^{-c_{3} y / \log y} d \tau \ll \sqrt{\frac{y}{\log y}} \int_{\sqrt{2}}^{\infty} \tau^{-c_{14} y / \log y} d \tau \ll \frac{\log y}{y} .
\end{aligned}
$$

This completes the proof.

\section{Proof of Theorem 3}

For each $y \geqslant 2$ fixed, we consider two functions of $u \in[1, \infty)$ :

$$
\alpha_{k, u}:=\alpha_{k}\left(y^{u}, y\right), \quad f(u):=\log \left(\frac{y^{u \alpha_{k, u}} \zeta\left(\alpha_{k, u}, y\right)^{k}}{\alpha_{k, u} \sqrt{2 \pi \phi_{2}\left(\alpha_{k, u}, y\right)}}\right) .
$$

Then Theorem 1 can be written as $S_{k}(x, y)=\exp \{f(u)+O(1 / \bar{u})\}$. Thus it suffices to show

$$
f(u+t)=f(u)+t \alpha_{k, u} \log y+O(1 / \bar{u}) \quad(u \geqslant 1,0 \leqslant t \leqslant 1) .
$$


For this we first write, for $u \geqslant 1$ and $0 \leqslant t \leqslant 1$,

$$
f(u+t)=f(u)+t f^{\prime}(u)+O\left(\sup _{0 \leqslant t \leqslant 1}\left|f^{\prime \prime}(u+t)\right|\right) .
$$

From the definition of $f(u)$, a simple calculation gives us

$$
\begin{aligned}
f^{\prime}(u)= & \alpha_{k, u} \log y-\frac{\alpha_{k, u}^{\prime}}{\alpha_{k, u}}-\frac{\phi_{3}\left(\alpha_{k, u}, y\right) \alpha_{k, u}^{\prime}}{2 \phi_{2}\left(\alpha_{k, u}, y\right)} \\
f^{\prime \prime}(u)= & \alpha_{k, u}^{\prime} \log y-\frac{\alpha_{k, u} \alpha_{k, u}^{\prime \prime}-\alpha_{k, u}^{\prime 2}}{\alpha_{k, u}^{2}}-\frac{\phi_{4}\left(\alpha_{k, u}, y\right) \alpha_{k, u}^{\prime 2}+\phi_{3}\left(\alpha_{k, u}, y\right) \alpha_{k, u}^{\prime \prime}}{2 \phi_{2}\left(\alpha_{k, u}, y\right)} \\
& -\frac{1}{2}\left(\frac{\phi_{3}\left(\alpha_{k, u}, y\right) \alpha_{k, u}^{\prime}}{\phi_{2}\left(\alpha_{k, u}, y\right)}\right)^{2} .
\end{aligned}
$$

where we have used the relation $u \log y=-\phi_{1}\left(\alpha_{k, u}, y\right)$ for simplifying. On differentiating the preceding equation with respect to $u$ and by using Lemma 2.3, it follows

$$
\alpha_{k, u}^{\prime}=-\frac{\log y}{\phi_{2}\left(\alpha_{k, u}, y\right)} \asymp \bar{u} /\left(u^{2} \log y\right), \quad \alpha_{k, u}^{\prime \prime}=-\frac{\phi_{3}\left(\alpha_{k, u}, y\right) \alpha_{k, u}^{\prime 2}}{\phi_{2}\left(\alpha_{k, u}, y\right)} \asymp \bar{u} /\left(u^{3} \log y\right) .
$$

From these estimations and (2.6)-(2.7) in Lemma 2.2, we easily deduce

$$
f^{\prime}(u)=\alpha_{k, u} \log y+O(1 / \bar{u}), \quad \quad f^{\prime \prime}(u) \ll 1 / \bar{u} .
$$

Inserting into (5.2) leads to the formula (5.1). This completes the proof.

\section{Proof of Theorem 4}

Similarly to Lemma 4.2 , we can prove the following result.

Lemma 6.1. For $y \geqslant y_{0}(\varepsilon, k)$ and $u \geqslant k / \log 2+\varepsilon$, we have

$$
\frac{1}{2 \pi i} \int_{\alpha_{k}-i / \log y}^{\alpha_{k}+i / \log y} \zeta(s, y)^{k} x^{s} d s=\frac{x^{\alpha_{k}} \zeta\left(\alpha_{k}, y\right)^{k}}{\sqrt{2 \pi \lambda_{2}}}\left\{1+O\left(\frac{1}{\bar{u}}\right)\right\} .
$$

Further the same formula also holds for

$$
\frac{1}{2 \pi} \int_{\alpha_{k}-i / \log y}^{\alpha_{k}+i / \log y}\left|\zeta(s, y)^{k} x^{s}\right||d s| .
$$

Clearly the desired result is trivial if $u \leqslant k / \log 2+\varepsilon$. Next we suppose $u \geqslant$ $k / \log 2+\varepsilon$. Put $x^{\prime}:=x+x / z$ and $\tilde{\alpha}_{k}:=\alpha_{k}\left(x^{\prime}, y\right)$. On differentiating the equation $-\phi_{1}\left(\alpha_{k}, y\right)=\log x$ with respect to $x$, it follows $\partial \alpha_{k}(x, y) / \partial x=-1 / x \lambda_{2}$. By using Lemma 2.3, we immediately see

$$
0<-\frac{\partial \alpha_{k}}{\partial x}(x, y) \asymp \frac{\bar{u}}{x(u \log y)^{2}}, \quad 0<\alpha_{k}-\tilde{\alpha}_{k} \ll \frac{\bar{u}}{z(u \log y)^{2}} \ll \frac{1}{u(\log y)^{2}} .
$$


From these we easily deduce, for $\tau \in \mathbb{R}$ and $\beta \in\left[\tilde{\alpha}_{k}, \alpha_{k}\right]$,

$$
x^{\prime \beta}|\zeta(\beta+i \tau, y)|^{k} \asymp x^{\alpha_{k}}\left|\zeta\left(\alpha_{k}+i \tau, y\right)\right|^{k} .
$$

Lemma 4.1 allows us to write

$$
\begin{aligned}
& S_{k}\left(x^{\prime}, y\right)=\frac{1}{2 \pi i} \int_{\tilde{\alpha}_{k}-i / \log y}^{\tilde{\alpha}_{k}+i / \log y} \zeta(s, y)^{k} \frac{x^{\prime s}}{s} d s+O\left(x^{\alpha_{k}} \zeta\left(\alpha_{k}, y\right)^{k} R_{0}(x, y)\right), \\
& S_{k}(x, y)=\frac{1}{2 \pi i} \int_{\alpha_{k}-i / \log y}^{\alpha_{k}+i / \log y} \zeta(s, y)^{k} \frac{x^{s}}{s} d s+O\left(x^{\alpha_{k}} \zeta\left(\alpha_{k}, y\right)^{k} R_{0}(x, y)\right),
\end{aligned}
$$

where $R_{0}(x, y):=Y_{\varepsilon}(y)^{-1}+e^{-c_{13} u /(\log u)^{2}}$ and we have used (6.1) with $\tau=0$ in the error term of (6.2). We deform the segment of integration $\left[\tilde{\alpha}_{k}-i / \log y, \tilde{\alpha}_{k}+i / \log y\right]$ into the line breaked $\tilde{\alpha}_{k}-i / \log y, \alpha_{k}-i / \log y, \alpha_{k}+i / \log y, \tilde{\alpha}_{k}+i / \log y$. With the help of (6.1) and the second inequality in Lemma 2.6, we easily see that the contribution of horizontal segments is

$$
\ll \frac{x^{\alpha_{k}}\left|\zeta\left(\alpha_{k} \pm i / \log y, y\right)\right|^{k}}{\alpha_{k}+1 / \log y}\left|\alpha_{k}-\tilde{\alpha}_{k}\right| \ll x^{\alpha_{k}} \zeta\left(\alpha_{k}, y\right)^{k} R_{0}(x, y) .
$$

According to the residue theorem, we obtain

$$
S_{k}\left(x^{\prime}, y\right)=\frac{1}{2 \pi i} \int_{\alpha_{k}-i / \log y}^{\alpha_{k}+i / \log y} \zeta(s, y)^{k} \frac{x^{\prime s}}{s} d s+O\left(x^{\alpha_{k}} \zeta\left(\alpha_{k}, y\right)^{k} R_{0}(x, y)\right) .
$$

Combining with (6.3), we deduce

$$
S_{k}\left(x^{\prime}, y\right)-S_{k}(x, y)=P_{k}(x, y)+O\left(x^{\alpha_{k}} \zeta\left(\alpha_{k}, y\right)^{k} R_{0}(x, y)\right),
$$

where

$$
P_{k}(x, y):=\frac{1}{2 \pi i} \int_{\alpha_{k}-i / \log y}^{\alpha_{k}+i / \log y} \zeta(s, y)^{k} x^{s} \frac{(1+1 / z)^{s}-1}{s} d s .
$$

Observing that

$$
\frac{(1+1 / z)^{s}-1}{s}=\frac{1}{z}+O\left(\frac{1}{z^{2}}\right) \quad(z \geqslant 1,|s| \ll 1)
$$

Lemma 6.1 and Theorem 1 imply

$$
P_{k}(x, y)=\frac{x^{\alpha_{k}} \zeta\left(\alpha_{k}, y\right)^{k}}{z \sqrt{2 \pi \lambda_{2}}}\left\{1+O\left(\frac{1}{\bar{u}}+\frac{1}{z}\right)\right\}=\frac{\alpha_{k}(x, y)}{z} S_{k}(x, y)\left\{1+O\left(\frac{1}{\bar{u}}+\frac{1}{z}\right)\right\}
$$

Finally by using Lemmas $2.2-2.3$ and Theorem 1 , we easily verify

$$
x^{\alpha_{k}} \zeta\left(\alpha_{k}, y\right)^{k} R_{0}(x, y) \ll S_{k}(x, y) \alpha_{k} \sqrt{\lambda_{2}} R_{0}(x, y) \ll S_{k}(x, y) R(x, y) .
$$

Now the desired result follows from (6.5), (6.6) and (6.7).

Acknowledgements. The author is grateful to Jie $\mathrm{Wu}$ for his help on the realization of this work, and to Hakim Smati for his comments on an earlier version of the paper. 


\section{References}

[1] A. Hildebrand, Integers free of large prime factors and the Riemann Hypothesis, Mathematika 31 (1984), 258-271.

[2] A. Hildebrand, On the number of positive integers $\leqslant x$ and free of prime factors $>y$, J. Number Theory 22 (1986), 289-307.

[3] A. Hildebrand and G. Tenenbaum, On integers free of large prime factors, Trans. Am. Math. Soc. 296 (1986), 265-290.

[4] A. Hildebrand and G. Tenenbaum, Integers without large prime factors, J. Théor. Nombres Bordeaux 5 (1993), 265-290.

[5] K. K. Norton, Numbers with small prime factors and least kth power non residue, Mem. Am. Math. Soc. 106 (1971).

[6] E. Saias, Sur le nombre des entiers sans grand facteur premier, J. Number Theory 32 (1989), 78-99.

[7] H. Smida, Sur les puissances de convolution de la fonction de Dickman, Acta Arith. 59 (1991), 123-143.

[8] H. Smida, Valeur moyenne des fonctions de Piltz sur les entiers sans grand facteur premier, Acta Arith. 63 (1993), 21-50.

[9] G. Tenenbaum, La méthode du col en théorie analytique des nombres, in: Séminaire de Théorie des Nombres (C. Goldstein, ed.), Paris 1985-86, Birkhäuser, Progress in Math. Vol. 75, 411-441.

[10] G. Tenenbaum, Introduction à la théorie analytique et probabiliste des nombres, Cours Spécialisés $\mathrm{N}^{\circ} 1$, Collection SMF, Société Mathématique de France, 1995.

[11] G. Tenenbaum, en collaboration avec J. Wu, Exercices corrigés de théorie analytique et probabiliste des nombres, Cours Spécialisés $\mathrm{N}^{\circ} 2$, Collection SMF, Société Mathématique de France, 1996.

[12] T.Z. Xuan, The average order of $d_{k}(n)$ over integers free of large prime factors, Acta Arith. 55 (1990), 249-260.

Département de Mathématiques

(Received 2003 2003)

Faculté des Sciences de Tunis

Université de Tunis II

1060 Tunis, Tunisie 